\title{
The Effect of a Short-Term Training Period on Physiological Parameters and Running Performance in Recreationally Active Female Runners
}

\author{
Irena Auersperger ${ }^{1}$, Iva Jurov ${ }^{1}$, Klemen Laurencak', Bojan Leskosek ${ }^{1}$ and Branko Skof ${ }^{1}$ \\ 'University of Ljubljana, Faculty of Sport, Ljubljana, Slovenia
}

\begin{abstract}
This paper aimed to analyse an individually set training programme in recreationally active female runners and its effects on endurance and performance. Nine female athletes (age 34.0 \pm 5.4 years) went through eight weeks of polarized training based on a modified training impulse method. Their training zones were established individually based on their heart rate measured with an incremental running test. Their programme was polarized to elicit positive changes in aerobic capacity and running performance in a $2400 \mathrm{~m}$ test. Their physiological parameters (VO2max, HR, respiratory compensation threshold) ventilation were measured in an incremental running test. The participants attended most of the training sessions and showed great motivation in their individually set training regime. We observed positive changes in all measured parameters (final treadmill velocity, distance covered on the treadmill, VO2max, the velocity at RCT) in an incremental test and better performance on the 2400 $m$ run. A modified TRIMP concept in an eight-week running programme is a valid method in prescribing training to recreationally active female runners. It elicits positive changes in performance and supports well-being and health.
\end{abstract}

Keywords: training periodization, TRIMP, VO2 max, running, monitoring training load

\section{Introduction}

Endurance training is widely known to contribute to better health and well-being (Ainsworth et al., 2011; Haskell et al., 2007; Marti, 1991; Samitz, Egger, \& Zwahlen, 2011). Running, in particular, is becoming increasingly popular as it is one of the most affordable forms of aerobic exercise that can produce results the fastest. The American College of Sports Medicine (Haskell et al., 2007) recommends moderate-intensity aerobic exercise for a minimum of $30 \mathrm{~min}$, five days a week, or vigorous-intensity aerobic physical activity for a minimum of $20 \mathrm{~min}$, three days a week, to all healthy adults aged 18-65 years. These guidelines emphasize training at an intensity close to the traditional lactate threshold (Haskell et al., 2007).

In recent years, training distribution has received attention, as it could be a determinant of endurance training impact. A proposed training pattern termed 'polarized endurance training' (PET) (Esteve-Lanao, Foster, Seiler, \& Lucia, 2007; Muñoz, Seiler, Bautista, España, \& Esteve-Lanao, 2014; Seiler \& Kjerland, 2006; Tnønessen et al., 2014) consists of two distinct intensity levels. About $80 \%$ of training volume is performed at a low-intensity level, and about $20 \%$ of training volume is high-intensity training (HIT), which is an intensity above the lactate threshold (LT). There are also other approaches to distributing intensity zones. A number of research studies have identified intensity zones based on ventilatory thresholds (VT) and their associated heart rate $(\mathrm{HR})$ values identified during the incremental test. Three training zones are defined as zone 1, being low-intensity exercise performed below the first VT; zone 2, moderately high-intensity

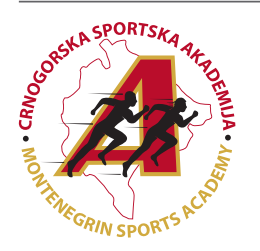

Correspondence:

I. Jurov

University of Ljubljana, Faculty of Sports, Gortanova 22, 1000 Ljubljana, Slovenia

E-mail: iva.jurov@fsp.uni-lj.si 
exercise in an intensity range between the VT and the respiratory compensation threshold (RCT); and zone 3, high-intensity aerobic exercise performed above the RCT (Muñoz et al., 2014). Taking into account both exercise volume and intensity into a single term, Banister et al. (Bannister, 1991) developed the concept of the training impulse (TRIMP). The original TRIMP method did not take into account the aforementioned intensity zones based on reference HR values. Foster et al. modified the original TRIMP concept by integrating total exercise volume and total intensity relative to three intensity zones (Foster et al., 2001; Foster, Rodriguez-Marroyo, \& Koning, 2017).

There is substantial evidence supporting highly trained endurance athletes from a variety of sports using PET pattern. It is not, however, yet evident whether this is also beneficial for recreational athletes performing a much smaller volume (i.e., 3-5 hours per week) and whether intensity distribution is crucial at all. A study from Muñoz et al. (2014) suggested that PET training can stimulate greater training effects compared with a programme at threshold range intensities in male recreational runners. PET has also shown benefits in raising VO2max compared to cross-fit endurance training in female runners (Carnes \& Mahoney, 2019). A recent systematic review of middle- and long-distance runners compared PET to pyramidal training and threshold training (Kenneally, Casado, \& Santos-Concejero, 2018). PET and pyramidal training appear to be more effective. However, only six intervention studies were suitable for the analysis, and other data were from three case studies and one review. Regarding these findings, further research is needed to support PET training advantages. Moreover, it would be beneficial to establish these recommendations in female recreational athletes.
Finally, using a running programme can be beneficial in delivering results in recreationally active individuals. Running related injuries are namely very common in recreational runners, especially in novice runners. They are an important factor of dropout from running. That is why running programmes can be effective not only in achieving greater performance but also in delivering greater health effects by achieving long term running participation (Kluitenberg et al., 2015).

The health benefits of exercise in recreationally active women are established (Marti, 1991), but little is known about improving performance and whether there are benefits of an individually determined training regime. The purpose of the study was to analyse the effectiveness of an eight-week individually set running intervention on the health and performance of nine female runners. The aim was to monitor and control the training load in order to ensure desired individually determined polarized endurance training.

\section{Methods \\ Subjects}

Nine healthy, active female recreational endurance runners were recruited to participate in the study. Prior to inclusion, all participants regularly had three to four regular running sessions per week, were non-smokers and were not taking any form of medication. The physical characteristics of the participants are shown in Table 1. Detailed history, physical examination, and laboratory analysis were performed before the study. All subjects were healthy at the start and during the research. The study protocol was approved by the National Ethical Committee of Slovenia. All participants gave written informed consent.

Table 1. Basic characteristics and body composition prior to training and post-study in the recovery phase

\begin{tabular}{cccccccccccc}
\hline & Age & $\begin{array}{c}\text { Body Height } \\
(\mathbf{c m})\end{array}$ & \multicolumn{2}{c}{ Body Mass $(\mathbf{k g})$} & \multicolumn{2}{c}{ BMI } & \multicolumn{2}{c}{$\%$ Fat } & \multicolumn{2}{c}{ Fat Free Mass (kg) } \\
& & & pre & post & pre & post & pre & post & pre & post \\
\hline M & 34.0 & 171.3 & 60.3 & 60.0 & 20.5 & 20.6 & 18.3 & 17.8 & 50.3 & 50.0 \\
SD & 5.4 & 6.8 & 6.9 & 7.1 & 1.8 & 2.0 & 2.8 & 2.6 & 4.8 & 4.5 \\
\hline
\end{tabular}

\section{Study design}

The study was completed during the intensified training phase (specific preparation), focusing on the $10 \mathrm{~km}(\mathrm{~N}=3)$ or the $21 \mathrm{~km}$ $(\mathrm{N}=6)$ run competition at the International Ljubljana Marathon in October 2008. The whole group completed a general preparation phase from April to August in that year. Before starting the specific training phase, all runners completed a two-week run-in period of low-intensity physical training to ensure familiarity with experimental procedures and to have reached a non-fatigued state. The physical training programme consisted of two three-week progressive overload periods (training loads Load1 and Load2), each followed by an easy week period (Easy Week 1 and Easy Week 2). After the second easy week, the runners participated in the race. This was followed by a recovery week. In the end, their physiological parameters were measured again. Table 2 shows the testing schedule for the study.

Table 2. Study protocol

\begin{tabular}{|c|c|c|c|c|c|c|c|c|c|c|}
\hline $\begin{array}{c}\text { General } \\
\text { Preparation } \\
\text { Period }\end{array}$ & \multicolumn{10}{|c|}{ Physical Training Program } \\
\hline week2 week1 & $\begin{array}{c}\text { week } \\
0\end{array}$ & $\begin{array}{c}\text { week } \\
1\end{array}$ & $\begin{array}{c}\text { week } \\
2\end{array}$ & $\begin{array}{c}\text { week } \\
3\end{array}$ & $\begin{array}{c}\text { week } \\
4\end{array}$ & $\begin{array}{c}\text { week } \\
5\end{array}$ & $\begin{array}{c}\text { week } \\
6\end{array}$ & $\begin{array}{c}\text { week } \\
7\end{array}$ & $\begin{array}{c}\text { week } \\
8\end{array}$ & $\begin{array}{c}\text { week } \\
9\end{array}$ \\
\hline \multirow[t]{4}{*}{$\begin{array}{l}\text { low-intensity } \\
\text { physical training }\end{array}$} & Baseline Testing & Trai & ining Loa & $\operatorname{ad} 1$ & $\begin{array}{c}\text { recovery } \\
\text { week } 1\end{array}$ & Trai & hing Loa & d 2 & $\begin{array}{c}\text { recovery } \\
\text { week } 2\end{array}$ & Post Study Testing \\
\hline & 1. IT & & & & & & & & & 2. IT \\
\hline & 1. RT & & & 2. RT & & 3. RT & & & 4. RT & 5. RT \\
\hline & anthropometry & & & & & & & & & anthropometry \\
\hline
\end{tabular}

Legend: IT-incremental test, RT-running test) 
Experimental procedures

\section{Running training}

The participants had four training sessions per week in training load phases consisting of one short aerobic interval training (at $88-95 \%$ maximum HR (HRmax)), one long aerobic interval training (up to $100 \%$ HRmax), an easy run (at $70-87 \%$ HRmax) of 6-8 km and a long-distance run (at 70$87 \%$ HRmax) $12-18 \mathrm{~km}$. Based on the runner's HRmax, which we determined during the incremental baseline test to exhaustion, we set each runner's training protocol to match the same training stimulus. The runners completed a $2400-\mathrm{m}$ time trial (Cooper) on an outdoor $400 \mathrm{~m}$ tartan track before commencing training and then every two to three weeks during the testing period. The programme was divided into two four-week training blocks, each consisting of three weeks of increasing load and one recovery week (Table 2). In the recovery period, interval training was replaced with an easy run of $6-8 \mathrm{~km}$. All training sessions were supervised by at least one qualified athlete coach and one member of the research group.

\section{Incremental test and anthropometry}

All runners completed an incremental test to exhaustion on a treadmill before the start and post-study. Each runner had previous experience with treadmill running and testing. After a six-min warm-up, an incremental protocol on a calibrated treadmill (Technogym, UK) with a $2 \%$ incline was performed. The starting velocity was $3 \mathrm{~km} / \mathrm{h}$ with speed increments of 2 $\mathrm{km} / \mathrm{h}$ every $2 \mathrm{~min}$. The runners walked the first stage and then ran until volitional exhaustion. The last half or full stage that the subject could sustain (for either $1 \mathrm{~min}$ or $2 \mathrm{~min}$ ) was defined as that individual's maximal speed. During recovery, the subjects walked at $5 \mathrm{~km} / \mathrm{h}$ for $5 \mathrm{~min}$. Respiratory parameters were assessed with a Cosmed K4b2 spirometry system (Rome, Italy) (McLaughlin, King, Howley, Bassett, \& Ainsworth, 2001). HR was recorded continuously during the test using telemetric heart monitors (Polar Electro, Oulu, Finland).

Anthropometric measurements were made before the start and post-study. Bodyweight $(\mathrm{kg})$ and height $(\mathrm{cm})$ were measured to the nearest $0.1 \mathrm{~kg}$ and $0.5 \mathrm{~cm}$, respectively, and body mass index (BMI) was calculated. Body fat percentage (\%) and lean mass $(\mathrm{kg})$ were assessed using the skinfold technique and calculated using Matiegka's method. Skinfold thicknesses at biceps, triceps, and subscapular were measured with GPM skinfold callipers (Siber Hegner \& Co. Ltd., Zurich, Switzerland) with a precision of $0.2 \mathrm{~mm}$.

\section{Quantification of training load}

Each participant wore a Polar $800 \mathrm{HR}$ monitor during the process to record training, not including warm-up and cooldown intervals. The following parameters were measured: total time spent in each intensity zone (zone 1, HR below the VT; zone 2, HR between VT and RCT; zone 3, HR above RCT) and total load (TRIMP score). Previous research on trained endurance athletes has shown that HR values at VT and RCT determined during laboratory testing remain stable over the season despite significant improvements in the workload eliciting both thresholds (Lucía, Hoyos, Perez, \& Chicharro JL., 2000).

We estimated the total exercise load (intensity $\times$ volume) using an approach to calculating the TRIMP based on Foster et al. (2001). This method uses HR data during exercise to integrate both total volume and intensity relative to three intensity zones. The score for each zone is computed by multiplying the accumulated duration in this zone by an intensity-weighted multiplier ( $1 \mathrm{~min}$ in zone 1 is given a score of $1,1 \mathrm{~min}$ in zone 2 is given a score of 2 , and $1 \mathrm{~min}$ in zone 3 is given a score of 3). Total TRIMP load is then obtained by summing the three zone scores.

\section{Data analysis}

Statistical analysis was performed with SPSS version 22 (IBM, Armonk, NY, USA) and Microsoft Excel (2016, Seattle, USA). A paired samples t-test investigated significant differences between methods. Data are reported as mean \pm standard deviation or percentage unless specified otherwise and participant characteristics pre- and post-study were compared.

\section{Results}

Baseline and post-study laboratory parameters

One of ten subjects was excluded from analysis due to incomplete training-data recording. Baseline and post-study parameters of body composition did not change significantly (Table 1).

\section{Quantification of training load}

None of the nine participants included in the study was injured during the training period (Auersperger idr., 2012). All of them performed $94 \%$ of the scheduled training sessions over the eight-week programme. The cumulative total duration of running training session over the experimental period (week 1-8) was $23.71 \pm 3.9$ hours and $235.79 \pm 39.16 \mathrm{~km}$. Other characteristics are represented in Table 3.

Table 3. Average training load and physiological parameters measured at incremental test after the study

\begin{tabular}{|c|c|c|c|c|c|c|c|}
\hline participant & $\begin{array}{c}\text { number of } \\
\text { sessions per } \\
\text { week }\end{array}$ & $\begin{array}{c}\text { duration of } \\
\text { training } \\
\text { (h/week) }\end{array}$ & $\begin{array}{c}\text { running } \\
\text { distance } \\
\text { (km/week) }\end{array}$ & $\begin{array}{c}\text { V02max } \\
\text { improvement }\end{array}$ & $\begin{array}{c}\text { VRCT } \\
\text { improvement }\end{array}$ & $\begin{array}{c}\text { final } \\
\text { treadmill } \\
\text { velocity }\end{array}$ & $\begin{array}{c}\text { distance } \\
\text { covered on } \\
\text { treadmill }\end{array}$ \\
\hline 1 & 30 & 2.7 & 34.3 & $5.51 \%$ & $5.66 \%$ & $4.76 \%$ & $6.53 \%$ \\
\hline 2 & 24 & 2.8 & 31.0 & $6.77 \%$ & $0.00 \%$ & $1.79 \%$ & $3.65 \%$ \\
\hline 3 & 18 & 3.4 & 35.1 & $6.76 \%$ & $8.89 \%$ & $7.02 \%$ & $14.53 \%$ \\
\hline 4 & 29 & 2.9 & 30.9 & $5.20 \%$ & $6.12 \%$ & $-3.17 \%$ & $0.03 \%$ \\
\hline 5 & 26 & 2.1 & 23.0 & $4.04 \%$ & $-5.66 \%$ & $3.64 \%$ & $7.83 \%$ \\
\hline 6 & 28 & 3.3 & 38.0 & $2.83 \%$ & $4.26 \%$ & $10.91 \%$ & $17.39 \%$ \\
\hline 7 & 26 & 2.9 & 31.0 & $9.33 \%$ & $3.77 \%$ & $-1.89 \%$ & $-6.10 \%$ \\
\hline 8 & 27 & 3.1 & 37.4 & $7.31 \%$ & $-2.13 \%$ & $1.64 \%$ & $2.78 \%$ \\
\hline 9 & 29 & 3.6 & 42.6 & $-0.56 \%$ & $8.51 \%$ & $0.00 \%$ & $-0.11 \%$ \\
\hline average & 26.33 & 3.0 & 33.7 & $5.24 \%$ & $3.27 \%$ & $2.68 \%$ & $5.11 \%$ \\
\hline
\end{tabular}

Legend: VRCT - velocity at respiratory compensation threshold 
The realization of the training programme is presented with modified TRIMP score in Figure 1.

\section{TRIMP SCORE}

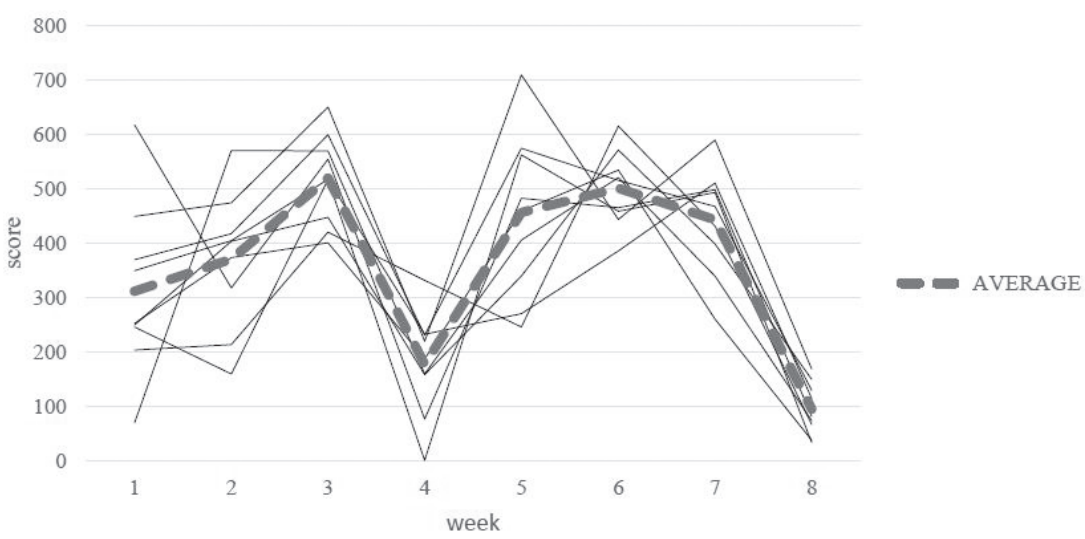

Figure 1. TRIMP score during 8-week training program

All but one runner improved their performance in the $2400 \mathrm{~m}$ test, as shown in Figure 2. We suspect her poorer per-

formance was due to the fact that she developed iron deficiency anaemia during the study (Auersperger idr., 2013).

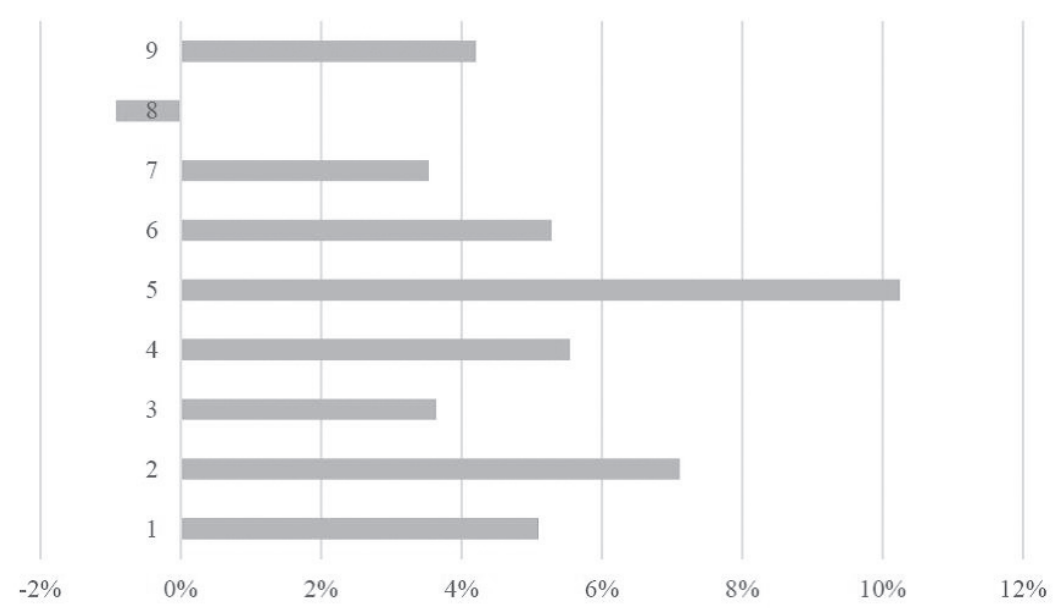

Figure 2. Individual performance improvement in $2400 \mathrm{~m}$ test conducted at week 9

Training intensity distribution (time spent in zones 1, 2 ent in the training protocol where the training load was mostly and 3) with training sessions per week for all athletes is prereduced by decreasing zone 3 and zone 2 . sented in Figure 3. It shows how two taper periods were pres-

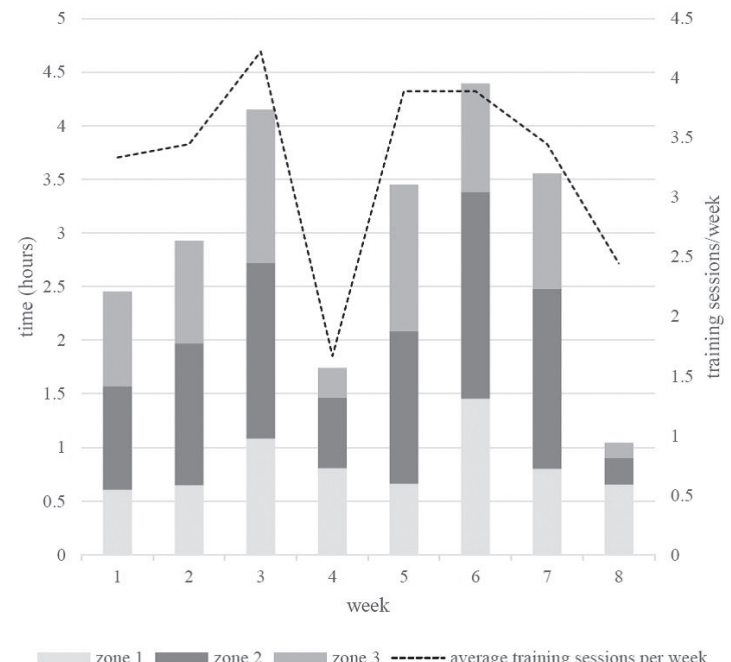

Figure 3. Exercise intensity and training unit distribution per week 
All of the participants completed the majority of the prescribed training programme. Training volume of $3.0 \pm 0.5 \mathrm{~h}$ per week $(0.8 \pm 0.3 \mathrm{~h}$ in zone $1,1.2 \pm 0.4 \mathrm{~h}$ in zone 2 and $0.9 \pm 0.4$ $\mathrm{h}$ in zone 3 ) including two taper periods in between elicited improvement not only in improvements of laboratory parameters but also of field test performance (Figure 2) was reported. We observed a tendency to improvement in running efficiency as the velocity at RCT increased on average by $3.27 \%(\mathrm{t}=1.94$, $\mathrm{p}=0.088)$. The sample size was not enough to prove statistically significant improvement, but the effect size is big (Cohen's $\mathrm{d}=0.65$ ). Additionally, we can conclude that participants' aerobic capacity increased in this relatively short time period as we measured a $5.2 \%$ increase in VO2max values $(t=5.40, p=0.001)$ (Table 3).

\section{Discussion}

This article presents how an individually set training load influences the performance of recreationally active female runners. Eight weeks of prescribed and supervised training with two taper periods elicited significant positive changes in participants' aerobic capacity, running efficiency and running time in a $2400 \mathrm{~m}$ test. This is in accordance with a study (Manzi, Iellamo, Impellizzeri, D’Ottavio, \& Castagna, 2009) conducted on recreationally active male long-distance runners where an individualized TRIMP concept was also used.

We expected an improvement in performance, as participants had been active before the study. On average, they performed running training two to three times weekly. This can also be shown also in their baseline $\mathrm{VO} 2 \mathrm{max}$ values which are above average for the general population $(35-40 \mathrm{ml} / \mathrm{min} / \mathrm{kg}$ for an average man (Guyton \& Hall, 2011)). We expected progress as we increased their training load (and TRIMP score) in this programme with special attention to their individual abilities that enabled the programme to be defined well. This is what enabled good compliance as the programme was not too intense. A study on 1419 recreational male and female runners showed that their average weekly running mileage is $47.3 \pm 17.5$ km (García-Pinillos, Ramírez-Campillo, Roche-Seruendo, Soto-Hermoso, \& Latorre-Román, 2019), which is above the average set for our participants. Our participants were females only, and we suggest that with average 33.7 weekly running distance the load on the runners was adequate and not too high.

Additionally, we expected better results as the programme had clearly defined sessions with supervision so that the runners were never alone in the process. The programme was set with the goal of improving VO2max since our initial aim was to increase aerobic capacity and its related positive health consequences (Lackland \& Voeks, 2014).

We used an incremental test to determine three intensity zones based on HR values. A study by Schumann et al. (Schumann, Botella, Karavirta, \& Häkkinen, 2017) used another approach in individualizing training intensity in thirty recreationally endurance-trained males. They used the Polar Training Load Feature and, compared to standardized endurance training, they did not find any differences in HR-derived indices. In contrast, our approach enabled runners to perform a session in a set zone since their HR was fixed in advance. This individualization of the programme made it possible not only to ascertain good cooperation and better improvement in an individual, but it also ensured good periodization and precise control over TRIMP. Consequently, we had good con- fidence that the TRIMP we set for our participants was also accomplished.

The programme was divided into two blocks of three weeks of higher load and one-week recovery. This is in accordance with a common knowledge used in endurance sports in which four-week mesocycles are regularly used since a ratio 3:1 between load and rest appeared to be efficient. Specifically, we planned extensive interval training in a way that every third run was performed uphill. This enabled the development of specific running power. Long aerobic interval training, which on average took 4-5 minutes, was also performed uphill in the last part of their entire training session. This was done with a goal of ensuring $\mathrm{VO} 2 \mathrm{max}$ values at the end of these sessions, as it is known this parameter can be influenced by intervals of 2-5 minutes (Daniels, 2005; Hill \& Rowell, 1997). With long runs, we aimed to indirectly influence $\mathrm{VO} 2$ max with maintaining aerobic endurance intensity levels that resulted in a 5.1\% longer distance achieved at the second incremental test (Table 3). We also observed a better running efficiency that resulted in a greater final treadmill velocity (Table 3 ). Short aerobic runs of $6-8 \mathrm{~km}$ were used for active recovery one to two days after the interval training session.

As observed from Figure 1, the TRIMP score did not reach its peak in Week 7 as expected in our programme. We observed fatigue in some participants that failed to complete all sessions in that week (hence $94 \%$ attendance instead of $100 \%$ ). We suggest this is another reason that a running programme with supervision and expertise is beneficial in improving performance. This caused our average TRIMP score to be less than predicted. However, overreaching with its possible negative consequences on the second incremental test and second $2400 \mathrm{~m}$ run was prevented. Self-reported subjective measures should be taken into monitoring as they provide significant insight into athletes well-being (Saw, Main, \& Gastin, 2016). Adjusting the programme to fatigue in these individuals also helped to avoid common running-related injuries (Hreljac, 2004). With regard to an improvement in all measured parameters, we can conclude that our goal of improving performance on the incremental test and the $2400 \mathrm{~m}$ running test was achieved. This indicates that an individualized running programme can be effective in running performance and well-being in recreationally active women. We also suggest this shows that our decision in not putting pressure on fatigued individuals to complete all sessions in Week 7 and mildly adjusting the programme could be the right decision.

After the training programme, the participants reported greater motivation than in their regular activities. Although this was not investigated with scientific methods or psychological questionnaires, we believe this contributed to a good attendance in determined sessions. This could be another advantage of a supervised running programme over a programme that is performed only by a runner alone.

We experienced some difficulties in controlling intensity in long-distance runs in which participants tended to run too fast (that is with an HR too high). We observed this when analysing HR values after their long-distance run sessions. If we perform this protocol again, we suggest monitoring HR values in real-time and adjusting their levels during the runs, if necessary. Secondly, as already emphasized, to ensure an ideal distribution of intensities in order to achieve a good anabolic and catabolic phase in our programme, Week 7 would need a greater TRIMP. This would be achieved if all prescribed ses- 
sions would have been performed. However, our participants were females that were not professional athletes. Their activities at the workplace and in their social environments also affected fatigue that aroused in some individuals at the end of our programme. This is why adjusting the programme is probably very realistic also in everyday practice when working with non-professional athletes.

We can conclude that an individually set modified TRIMP concept was beneficial in recreationally active female runners in our eight-week programme. It positively affected aerobic capacity and endurance performance. It also successfully avoided running-related injuries and supported well-being

\section{Acknowledgements}

There are no acknowledgements.

\section{Conflict of Interest}

The authors declare that there are no conflicts of interest.

Received: 27 August 2019| Accepted: 17 December 2019 | Published: 01 February 2020

\section{References}

Ainsworth, B. E., Haskell, W. L., Herrmann, S. D., Meckes, N., Bassett, D. R. J., Tudor-Locke, C., ... Leon, A. S. (2011). 2011 Compendium of Physica Activities: a second update of codes and MET values. Medicine \& Science in Sports \& Exercise, 43, 1575-1581. https://doi.org/10.1249/MSS.ObO

Auersperger, I., Knap, B., Jerin, A., Blagus, R., Lainščak, M., Skitek, M., \& Škof, B. (2012). The Effects of 8 Weeks of Endurance Running on Hepcidin Concentrations, Inflammatory Parameters, and Iron Status in Female Runners The Effects of 8 Weeks of Endurance Running on Hepcidin Concentrations , Inflammatory Parameters ,. International Journal of Sport Nutrition and Exercise Metabolism, 22, 55-63.

Auersperger, I., Škof, B., Leskošek, B., Knap, B., Jerin, A., \& Lainscak, M. (2013). Exercise-Induced Changes in Iron Status and Hepcidin Response in Female Runners. PLOS ONE, 8(3). https://doi.org/10.1371/journal. pone.0058090

Bannister, E. W. (1991). Modeling elite athletic performance. (W. H. Green H McDougal J, Ur.), Physiological Testing of High-Performance Athletes. Champaign: Human Kinetics.

Carnes, A. J., \& Mahoney, S. E. (2019). Polarized Versus High-Intensity Multimodal Training in Recreational Runners. International Journal of Sports Physiology and Performance, 14(1), 105-112. https://doi.org/10.1123/ ijspp.2018-0040

Daniels, J. (2005). Daniels' running formula (2nd izd.). Champaign, IL: Human Kinetics.

Esteve-Lanao, J., Foster, C., Seiler, S., \& Lucia, A. (2007). Impact of training intensity distribution on performance in endurance athletes. Journal of Strength and Conditioning Research, 21(3), 943-949. https://doi. org/10.1519/R-19725.1

Foster, C., Florhaug, J. a, Franklin, J., Gottschall, L., Hrovatin, L. a, Parker, S., .. Dodge, C. (2001). A new approach to monitoring exercise training. Journal of strength and conditioning research, 15(1), 109-115. https://doi.org /10.1519/1533-4287(2001)015<0109:ANATME>2.0.CO;2

Foster, C., Rodriguez-Marroyo, J. A., \& Koning, J. J. De. (2017). Monitoring Training Loads:The Past, the Present, and the Future. International Journal of Sports Physiology and Performance, 12, 2-8.

García-Pinillos, F., Ramírez-Campillo, R., Roche-Seruendo, L. E., Soto-Hermoso, V. M., \& Latorre-Román, P. Á. (2019). How do recreational endurance runners warm-up and cool-down? A descriptive study on the use of continuous runs. International Journal of Performance Analysis in Sport, 19(1), 102-109. https://doi.org/10.1080/24748668.2019.1566846

Guyton, A., \& Hall, J. E. (2011). Textbook of Medical Physiology, 12th Ed.

Haskell, W. L., Lee, I. M., Pate, R. R., Powell, K. E., Blair, S. N., Franklin, B. A., ... Bauman, A. (2007). Physical activity and public health: Updated recommendation for adults from the American College of Sports Medicine and the American Heart Association. Circulation, 116(9), 1081-1093. https://doi.org/10.1161/CIRCULATIONAHA.107.185649

Hill, D. W., \& Rowell, A. L. (1997). Responses to exercise at the velocity asso- and health. We suggest that if a running programme is supervised and individually determined, it has a greater potential in improving performance and supporting well-being in recreationally active females. It could also enable participants to adhere to physical activity guidelines with less chance of running-related injuries.

\section{Limitations}

Our study recruited only a moderate number of subjects; thus, we may not have been able to detect more subtle differences in measured parameters. Our results may not be applicable to men and should also be confirmed in elite athletes.

ciated with VO2max. Medicine and Science in Sports and Exercise, 29(1), 113-116.

Hreljac, A. (2004). Impact and Overuse Injuries in Runners. Medicine \& Science in Sports \& Exercise, 36(5), 845-849. https://doi.org/10.1249/01. MSS.0000126803.66636.DD

Kenneally, M., Casado, A., \& Santos-Concejero, J. (2018). The Effect of Periodization and Training Intensity Distribution on Middle- and Long-Distance Running Performance: A Systematic Review. International Journal of Sports Physiology and Performance, 13(9), 1114-1121. https://doi. org/10.1123/ijspp.2017-0327

Kluitenberg, B., van Middelkoop, M., Smits, D. W., Verhagen, E., Hartgens, F., Diercks, R., \& van der Worp, H. (2015). The NLstart2run study: Incidence and risk factors of running-related injuries in novice runners. Scandinavian Journal of Medicine \& Science in Sports, 25(5), e515-e523. https:// doi.org/10.1111/sms.12346

Lackland, D. T. \& Voeks, J. H. (2014). Metabolic Syndrome and Hypertension: Regular Exercise as Part of Lifestyle Management. Current Hypertension Reports, 16(11), 492. https://doi.org/10.1007/s11906-014-0492-2

Lucía, A., Hoyos, J., Perez, M., \& Chicharro JL. (2000). Heart rate and performance parameters in elite cyclists: a longitudinal study. Medicine and Science in Sports and Exercise, 32(10), 1777-1782.

Manzi, V., lellamo, F., Impellizzeri, F., D'Ottavio, S., \& Castagna, C. (2009). Relation between individualized training impulses and performance in distance runners. Medicine and Science in Sports and Exercise, 41(11) 2090-2096. https://doi.org/10.1249/MSS.0b013e3181a6a959

Marti, B. (1991). Health effects of recreational running in women. Some epidemiological and preventive aspects. Sports Medicine, 11(1), 20-51.

McLaughlin, J. E., King, G. A., Howley, E. T., Bassett, D. R. J., \& Ainsworth, B. E. (2001). Validation of the Cosmed K4b2 Portable Metabolic Measurement System. International Journal of Sports Medicine, 22, 280-284. https://doi.org/10.1097/00005768-200105001-01691

Muñoz, I., Seiler, S., Bautista, J., España, J., \& Esteve-Lanao, J. (2014). Does Polarized Training Improve Performance in Recreational Runners? International Journal of Sports Physiology and Performance, 9, 265-272. https:// doi.org/10.1123/IJSPP.2012-0350

Samitz, G., Egger, M., \& Zwahlen, M. (2011). Domains of physical activity and all-cause mortality: Systematic review and dose-response meta-analysis of cohort studies. International Journal of Epidemiology, 40(5), 1382 1400. https://doi.org/10.1093/ije/dyr112

Saw, A. E., Main, L. C., \& Gastin, P. B. (2016). Monitoring the athlete training response: subjective self-reported measures trump commonly used objective measures: a systematic review. British Journal of Sports Medicine, 50, 281-291. https://doi.org/10.1136/bjsports-2015-094758

Schumann, M., Botella, J., Karavirta, L., \& Häkkinen, K. (2017). Training-Load-Guided vs Standardized Endurance Training in Recreational Runners. International Journal of Sports Physiology and Performance, 12(3), 295-303. https://doi.org/10.1123/ijspp.2016-0093

Seiler, K. S., \& Kjerland, G. Ø. (2006). Quantifying training intensity distribution in elite endurance athletes: Is there evidence for an "optimal" distribution? Scandinavian Journal of Medicine and Science in Sports, 16(1), 49-56. https://doi.org/10.1111/j.1600-0838.2004.00418.x

Tnønessen, E., Sylta, Ø., Haugen, T. A., Hem, E., Svendsen, I. S., \& Seiler, S. (2014). The road to gold: Training and peaking characteristics in the year prior to a gold medal endurance performance. PLOS ONE, 9(7), 15-17. https://doi.org/10.1371/journal.pone.0101796 